\title{
EVALUACIÓN DE LAS SUBPOBLACIONES DE LINFOCITOS T EN PACIENTES TUBERCULOSOS EMPLEANDO LA MODULACIÓN CON TEOFILINA
}

\author{
Diana DLUGovitzKY (1), K. HUBER (2), G. WELKER (3) \& O. MOLTENI (4)
}

\section{RESUMEN}

Se evaluaron las poblaciones y subpoblaciones linfocitarias en pacientes con tuberculosis pulmonar antes y durante la terapia relacionanc.o estos valores con la incidencia y evolución de la enfermedad.

Pacientes en sus diversas manifestaciones clínicas, vírgenes de tratamiento, se estudiaron por baciloscopía (BAAR), radiología, i.d.r. Mantoux y análisis complementarios.

Se cuantificaron mediante la prueba de Rosetas espontáneas (RE) linfocitos $\mathrm{T}$ totales y activos $\left(\mathrm{RE}\right.$ a $4^{\circ} \mathrm{C}$ y $37^{\circ} \mathrm{C}$ ), $\mathrm{T}$ colaboradores (RE Teofilina Resistentes: RETR) y supresores (RE Teofilina Sensibles: RETS). Los exámenes se repitieron en los mismos sujetos iniciado el tratamiento y en testigos sanos (TS).

Se demostró en los pacientes en todas sus formas clínicas un descenso significativo en los valores relativos y absolutos de células $\mathrm{T}$ y en la relación RETR/RETS (menor de 1). Existe asociación entre la forma clínica y el número de linfocitos $T$ colaboradores.

Los pacientes en tratamiento con evolución favorable, evidenciaron un incremento significativo en los linfocitos $T$ totales, activos, colaboradores y en la relación RETR/RETS.

Los enfermos con baciloscopía altamente positiva presentaron i.d.r. Mantoux baja o negativa y marcado descenso de células inmunocompetentes. Se comprobó asociación entre estas tres variables, lo mismo que entre el estado nutricional y la predisposición a contraer la enfermedad.

UNITERMOS: Tuberculosis; Subpoblaciones de linfocitos T; Teofilina.

\section{INTRODUCCIÓN}

La tuberculosis es una compleja interacción entre el sistema inmune del huésped y el parásito intracelular Mycobacterium tuberculosis ${ }^{1,}$ 5, 8. 7. 10. 13. 24. 28 . Este bacilo ejerce una miríada de efec- tos sobre el sistema inmune y fagocitico mononuclear, ya que puede funcionar provocando fenómenos inflamatorios e infecciosos severos, o bien actuar como adyuvante estimulando o alterna ti-

(1) Investigadora del Consejo de Investigaciones de la U.N.R. Docente de la Latedra de Microbiología, a cargo de la Sección Inmunología. Facultad de Ciencias Médicas. Universidad Nacional de Rosario. Rosario, Argentina.

(2) Docente e Investigadora de la Cátedra de Microbiologia. Facultad de Ciencias Médicas. Universidad Nacional de Rosario. Rosario, Argentina.

(3) Médico del Hospital de Enfermedades Infecciosas V. L. Carrasco. Docente Cátedra de Enfermedades Infecciosas. Facultad de Ciencias Médicas. Universidad Nacional de Pocario. Posaric, Argentica.

(4) Profesor Titular de la Cátedra de Microbiologia, Parasitología y Virología. Facultad de Ciencias Médicas. Universidad Nacional de Rosario. Rosario, Argentina.

Dirección para la Correspondencia: Dra. Diana Dlugovitzky. Maipú 1340 - P. Baja Dto. "D". 2000 Rosario - Argentina. 
DLUGOVITZKY, D.; HUBER, K.; WELKER, G. \& MOLTENI, O. - Evaluación de las subpoblaciones de linfocitos $T$ en pacientes tuberculosos empleando la modulación con teofilina. Rev. Inst. Med. trop. S. Paulo, 33(2): 105-113, 1991.

vamente deprimiendo la respuesta inmune $\mathrm{i}^{1,10,17}$. Se acepta universalmente que los fenómenos in munológicos desempeñan un papel fundamental entre los mecanismos involucrados en la defensa contra el bacilo de $\operatorname{Koch}^{3,}$ 7, 24, 28, 29 .

Se han estudiado diversos aspectos de gran importancia para la comprensión de las complejas interacciones entre el Mycobacterium tuberculosis y el huésped infectado tales como regulación genética y los marcadores fenotípicos de los linfocitos $\mathrm{T}$ humanos, la naturaleza de los receptores antigénicos en las células $\mathrm{T}$, el procesamiento y presentación antigénica, las interacciones celulares que facilitan la adhesión molecular, así como las moléculas de superficie en las células fagocíticas que favorecen el ingreso de los patógenos a las células ${ }^{2,15,18,21,30}$ aunque interesa conocer aún más las alteraciones provocadas por el desequilibrio de las células inmunocomprometidas. Por ello la evaluación de las células que intervienen en la respuesta inmune celular, particularmente en las reacciones de hipersensibilidad retardada que se desencadenan en los pacientes tuberculosos no sólo nos permite in terpretar la patogénesis de la enfermedad, sino explicar ciertos fenómenos relacionados con la evolución de los pacientes ${ }^{8,18,22}$ y su capacidad para responder a la terapia.

Por otra parte, es fundamental la interacción reguladora de las diversas poblaciones celulares que se generan ante una infección tuberculosa, responsables de la respuesta inmune del huésped, produciendo efectos tanto protectores como patógenos para el huésped.

En esta experiencia se estimaron las pobla ciones linfocitarias de sangre periferica y las subpoblaciones funcionalmente distintas (linfocitos colaboradores y supresores) a fin de determinar si existe una correlación entre los niveles de células inmunocompetentes con las diversas manifestaciones clínicas de la enfermedad y con las diferentes formas evolutivas mediante la modulación con teofilina.

El advenimiento de los anticuerpos monoclonales dirigidos hacia subclases de linfocitos $T$ ha permitido en algunas experiencias cuantificar estas subpoblaciones. LIMANIBUL $Y$ SHOAT ${ }^{19,27}$ demostraron que las células Teofi- lina Resistentes y Sensibles con receptores Fc de la IgM, constituyen células "helper" o colaboradoras y supresoras que se corresponden a las subpoblaciones CD4 y CD8 respectivamente. Sin embargo las diferencias en los receptores de aquellas subpoblaciones (detectadas por su sen sibilidad a la Teofilina) determina una mayor heterogeneidad de las mismas con respecto a CD4 y CD8. Por ello la aplicación de la técnica de LIMANIBUL no estima exactamente a estas últimas. Aún así esta metodología ha permitido cuantificar con cierta aproximación las diferentes subpoblaciones en los pacientes tuberculosos mediante una técnica accesible que podría aplicarse sin dificultad al seguimiento de los enfermos en los servicios asistenciales de mediana complejidad.

\section{MATERIAL Y METODOS}

Pacientes: Se trabajó con pacientes tuberculosos con localización pulmonar, de ambos sexos y edades que oscilaron entre los 16 y 72 años, vírgenes de tratamiento, con baciloscopia positiva (BAAR: +) a los que se les efectuó exámenes clínicos, radiológicos, intradermo reacción de Mantoux (i. d. M) con PPD 2 U.T. y otros exámenes de laboratorio complementarios (Hemograma, Eritrosedimentación, Glicemia, Uremia) además de la confirmación del diagnóstico mediante cultivo en medio de Lowenstein Jensen.

De acuerdo a los datos obtenidos, los casos de tuberculosis fueron clasificados en relación a sus imágenes radiológicas, i. d. M y manifestaciones clínicas en: Leve (L), Moderadamente Avanzada (MA), Avanzada (A) y Grave (G).

El estudio se repitió en los pacientes bajo tratamiento mensualmente durante los cuatro meses después de iniciada la quimioterapia. Simultáneamente se siguió la evolución de los enfermos a través de controles clínicos, radiológico y baciloscópico.

Todos los exámenes y determinaciones efectuados a los pacientes también se realizaron con un grupo testigo o control constituído por individuos sanos (TS) de ambos sexos y edades variables de la misma condición socio-económica de los enfermos. En este grupo testigo, se incluyeron algunos sujetos aleatoriamente (Testigos po- 
DLUGOVITZKY, D.; HUBER, K.; WELKER, G. \& MOLTENI, O. - Evaluación de las subpoblaciones de linfocitos $T$ en pacientes tuberculosos empleando la modulación con teofilina. Rev. Inst. Med. trop. S. Paulo, 33(2): 105-113, 1991.

blacionales) y también familiares o personas que convivían con los enfermos (Testigos convivientes sanos).

Determinación de células inmunocompetentes: Se cuantificaron los niveles de linfocitos $T$ y las subpoblaciones de los mismos, $T$ colaboradores y T supresores ${ }^{4,31,32}$. Se practicó la prueba de hemocitoadherencia (Prueba de Rosetas espontáneas) que permite estimar linfocitos $T$ totales y linfocitos $\mathrm{T}$ activos y una modificación de esta técnica basada en la sensibilidad a la teofilina ${ }^{18,27}$, determinando por este método linfocitos $\mathrm{T}$ colaboradores y supresores.

Prueba de hemocitoadherencia: En esta têcnica la habilidad de los linfocitos $T$ para formar rosetas espontáneas uniendo eritrocitos de carnero a su superficie es usada como marcador para tales células.

Se obtuvieron muestras de sangue periférica heparinizada de donde se separaron los linfocitos a través de un gradiente de Ficol-Hypaque, centrifugando a $400 \mathrm{~g}$ durante 45 minutos. Los linfocitos fueron resuspendidos en solución reguladora de fosfato (Phosphate Buffer saline: PBS) hasta lograr una concentración de $3.10^{6} \mathrm{cell} / \mathrm{ml}$.

Glóbulos rojos de carnero (GRC): se obtuvieron por punción venosa $\mathrm{y}$ se conservaron en solución de Alsever al 50\%. Separados los GRC se lavaron 3 veces y resuspendieron en PBS al $0.5 \%$.

Rosetas E Activas (REA): En cada prueba se incubó $0.20 \mathrm{ml}$ de la suspensión de linfocitos con $0.20 \mathrm{ml}$ de la suspensión de GRC en un baño a $37^{\circ} \mathrm{C}$ durante 5 minutos y se centrifugó a 400 g durante 5'. Se evaluaron al microscopio las células formadoras de rosetas, contando 200 linfocitos y las células que hubieran adherido dos o más GRC se consideraron linfocitos $\mathrm{T}$.

Rosetas E Totales (RET): Se procedió en la misma forma que en la determinación de Rosetas $\mathrm{E}$ activas, pero luego de la incubación a $37^{\circ} \mathrm{C}$ y centrifugación posterior, se procedió a incubar la mezcla a $4^{\circ} \mathrm{C}$ durante $60^{\prime}$. La cuantificación microscópica permitió estimar los linfocitos T totales.
Rosetas E teofilina Resistentes (RETR): A través de la incubación de la suspensión de linfocitos, separados previamente a través del gradiente de Ficoll-Hypaque, con solución de Teofilina $5 \mathrm{mM}$ durante una hora, se separaron las subpoblaciones de linfocitos $\mathrm{T}$ colaboradores (Teofilina resistentes) y supresores (Teofilina sensibles) que luego se cuantificaron por la Prueba de Rosetas espontáneas a $4^{\circ} \mathrm{C}$ y $37^{\circ} \mathrm{C}$ antes descripta $^{19,27}$.

Análisis estadísticos: Se compararon los valores de Rosetas E y Rosetas E Teofilina Resistentes tanto totales (a $4^{\circ} \mathrm{C}$ ) como activas (a $37^{\circ} \mathrm{C}$ ) en ambos casos de los pacientes tuberculosos en sus diversas formas clínicas y del grupo TS, y también los datos de dichas determinaciones de los pacientes no tratados y de los mismos después de 4 meses de tratamiento. Teniendo en cuenta que los datos porcentuales de Rosetas no presentan una distribución normal, se trans formaron obteniendo los valores de arco seno y se analizaron mediante el test de medias. Se evaluó la relación entre los niveles de Rosetas E (RE) y Rosetas E Teofilina Resistentes (RETR), en ambos casos tan to totales $\left(4^{\circ} \mathrm{C}\right)$ como activas $\left(37^{\circ} \mathrm{C}\right)$ y las diferentes manifestaciones clínicas. Para ello se confeccionaron tablas de contingencia, relacionando ambas variables y aplicando luego el test de independencia de $\boldsymbol{\chi}^{2}$.

Por otra parte, las diferencias entre los valores absolutos de linfocitos $\mathrm{T}$ (obtenidos indirectamente a través de la determinación de Rosetas E y E activas, glóbulos blancos y fórmula leucocitaria del paciente) entre distintos estadíos de TBC y los del grupo TS, se analizaron mediante el test de $\mathrm{t}$ Student.

Aplicando el test de asociación de $\chi^{2}$ se estudió la relación entre baciloscopía (BAAR), estadíos de la TBC y respuesta a la i.d.r. de Mantoux.

También se comparó por el test de medias la relación entre el valor del cociente RETR/ RETS en distintas formas clínicas de la TBC con la de los TS. La asociación entre las manifestaciones clínicas de la enfermedad y estado nutricional se analizó por $\boldsymbol{\chi}^{2}$.

\section{RESULTADOS}

Los resultados indican que los pacientes tu berculosos en todas las manifestaciones clínicas 
DLUGOVITZKY, D.; HUBER, K.; WELKER, G. \& MOLTENI, O. - Evaluación de las subpoblaciones de linfocitos $T$ en pacientes tuberculosos empleando la modulación con teofilina. Rev. Inst. Med. trop. S. Paulo, 33(2): 105-113, 1991.

de la enfermedad (L, MA, A, G) presentan un descenso en los niveles de células inmunocompetentes con respecto a los sujetos sanos, en sus valores porcentuales y absolutos. Se determinó en los pacientes vírgenes de tratamien to una disminución en los niveles de Rosetas $\mathrm{E}$ totales (RE a $4^{\circ} \mathrm{C}$ ) y $\mathrm{E}$ activas $\left(\mathrm{RE}\right.$ a $37^{\circ} \mathrm{C}$ ) con respecto al grupo control o TS $(\mathrm{p}<0,01)$.

Los valores absolutos de linfocitos T totales/ $\mathrm{mm}^{3}$ y activos calculados con los correspondien tes datos de Rosetas, glóbulos blancos y fórmula leucocitaria, también se hallaron significativamente disminuídos $(\mathrm{p}<0.01)$.

Las estimaciones de las poblaciones linfocitarias en su conjunto no difirieron significativamente entre los grupos de pacientes con diferentes estados clínicos de tuberculosis, pero se verificó mediante pruebas estadísticas una fuerte asociación entre tales manifestaciones clínicas y el número de RETR.

La relación RETR/RETS que equivale a T colaboradoras/T supresoras fue menor a 1 en los pacientes no tratados y significativamente inferior a la determinada en el grupo TS $(p<0.01)$.

La marcada depresión de células inmunocompetentes de los pacientes antes del trata- miento se modificó después de comenzado el mismo y a los 4 meses de su inicio se observó, particularmente en los enfermos con evolución favorable, un restablecimiento de los linfocitos $T$ totales, activos y colaboradores (RET, REA, RETR a $4^{\circ} \mathrm{C}$ y $37^{\circ} \mathrm{C}$ ). Las Tablas 1 y 2 resumen los resultados obtenidos en estos pacientes antes y después de iniciado el tratamiento, que difirieron significativamente $(p<0.01)$.

También se registró un aumento en la relación RETR/RETS cuyo valor fue superior a 1 en los sujetos tratados a excepción de aquellos pacientes con tuberculosis grave. La relación RETR/RETS fue más elevada en los pacientes sometidos a quimioterapia a la de estos sujetos antes de iniciar el tratamiento $(\mathrm{p}<0.01)$ Tabla 3. El valor promedio del cociente RETR/RETS determinado en individuos normales (Testigos sanos) es 1.15 inferior al del cociente CD4/CD8 $=2$, obtenido utilizando anticuerpos monoclonales.

Tal diferencia obedece según lo afirmado anteriormente, a que las poblacions RETR/RETS evaluadas por el método de Rosetas + Teofilina, son heterogéneas con respecto a las poblaciones CD4 y CD8 y por ello la técnica empleada en este trabajo no detecta exactamente CD4/CD8.

TABLA 1

Celulas inmunocompetentes (C.I.) evaluadas por el test de rosetas totales activas y sensibles a la teofilina en pacientes tuberculosos virgenes de tratamiento y testigos sanos.

\begin{tabular}{|c|c|c|c|c|c|c|c|c|}
\hline C. & Linfocitos & $\mathrm{ST}$ totales & Linfocitos & T activos & & & & \\
\hline Cond & $\begin{array}{c}\mathrm{RET}\left(4^{\circ} \mathrm{C}\right) \\
\%\end{array}$ & Valor absoluto & $\underset{\%}{\mathrm{RET}\left(37^{\circ} \mathrm{C}\right)}$ & Valor absoluto & $\begin{array}{l}\text { RETR } \\
4^{\circ} \mathrm{C}(\%)\end{array}$ & $\begin{array}{c}\text { RETR } \\
37^{\circ} \mathrm{C}(\%)\end{array}$ & $\begin{array}{c}\text { RETS } \\
4^{\circ} \mathrm{C}(\%)\end{array}$ & $\begin{array}{c}\text { RETS } \\
37^{\circ} \mathrm{C}(\%)\end{array}$ \\
\hline $\begin{array}{c}\mathbf{L} \\
\mathbf{n}=17\end{array}$ & $37^{*} \pm 15.36$ & $1004^{*} \pm 313.8$ & $14.5^{*} \pm 7.1$ & $622^{*} \pm 207.4$ & $18.2^{*} \pm 7.04$ & $7^{*} \pm 3.86$ & $18.8 \pm 8.34$ & $7.5 \pm 2.76$ \\
\hline $\begin{array}{c}\text { MA } \\
\mathrm{n}=39\end{array}$ & $34.8^{*} \pm 10.46$ & $983^{*} \pm 428.4$ & $19.3^{*} \pm 6.24$ & $476^{*} \pm 184.1$ & $13.7^{*} \pm 9$ & $8.2^{*} \pm 7.97$ & $21.1 \pm 1.46$ & $11.1 \pm 8.1$ \\
\hline $\begin{array}{c}\text { A } \\
\mathbf{n}=36\end{array}$ & $33.8^{*} \pm 11.32$ & $938^{*} \pm 330$ & $17.7^{*} \pm 6.76$ & $434 * \pm 130.91$ & $15.6^{*} \pm 10.78$ & $5.5^{*} \pm 5.11$ & $18.2 \pm 6.9$ & $12.2 \pm 5.4$ \\
\hline $\begin{array}{c}\mathrm{G} \\
\mathrm{n}=18\end{array}$ & $34.9^{*} \pm 9.05$ & $734^{*} \pm 189.6$ & $18.7^{*} \pm 8.71$ & $330^{*} \pm 98.2$ & $12.2^{*} \pm 6.01$ & $7.8^{*} \pm 4.43$ & $22.7 \pm 7.8$ & $10.9 \pm 6.4$ \\
\hline $\begin{array}{c}\mathrm{TS} \\
\mathrm{n}=15\end{array}$ & $64^{*} \pm 8.07$ & $1874^{*} \pm 503.7$ & $29.93 * \pm 5.34$ & $1078^{*} \pm 327.4$ & $28.9^{*} \pm 6.2$ & $15.06^{*} \pm 4.32$ & $25.1 \pm 6.7$ & $12.87 \pm 4.86$ \\
\hline
\end{tabular}

$\overline{\mathrm{x}} \pm \mathrm{s}$ : média aritmética \pm desvio standar; TBC: L: leve; MA: moderadamente avanzada; A: avanzada; G: grave; TS: testigos sanos; RET: rosetas E totales; REA: rosetas E activas; RETR: rosetas E teofilina resistentes; RETS: rosetas E teofilina sensibles; ${ }^{*} \operatorname{RET}\left(4^{\circ} \mathrm{C}\right), \operatorname{REA}\left(37^{\circ} \mathrm{C}\right)$, RETR $\left(4^{\circ} \mathrm{C}\right.$ y $\left.37^{\circ} \mathrm{C}\right)$ significativamente menores que los correspondientes valores de T'S $\mathrm{p}<0,01$. 
DLUGOVITZKY, D.; HUBER, K.; WELKER, G. \& MOLTENI, O. - Evaluación de las subpoblaciones de linfocitos T en pacientes tuberculosos empleando la modulación con teofilina. Rev. Inst. Med. trop. S. Paulo, 33(2): 105-113, 1991.

TABLA 2

Celulas inmunocompetentes (C.I.) evaluadas por el test de rosetas totales activas y sensibles a la teofilina en pacientes tuberculosos tratados.

\begin{tabular}{|c|c|c|c|c|c|c|c|c|}
\hline & \multicolumn{2}{|c|}{ Linfocitos $\mathrm{T}$ totales } & \multicolumn{2}{|c|}{ Linfocitos $T$ activos } & \multirow[b]{2}{*}{$\begin{array}{l}\text { RETR } \\
4^{\circ} \mathrm{C}(\%)\end{array}$} & \multirow[b]{2}{*}{$\begin{array}{c}\text { RETR } \\
37^{\circ} \mathrm{C}(\%)\end{array}$} & \multirow[b]{2}{*}{$\begin{array}{c}\text { RETS } \\
4^{\circ} \mathrm{C}(\%)\end{array}$} & \multirow[b]{2}{*}{$\begin{array}{c}\text { RETS } \\
37^{\circ} \mathrm{C}(\%)\end{array}$} \\
\hline Co & $\underset{\%}{\operatorname{RET}\left(4^{\circ} \mathrm{C}\right)}$ & Valor absoluto & $\underset{\%}{\operatorname{RET}\left(37^{\circ} \mathrm{C}\right)}$ & Valor absoluto & & & & \\
\hline $\begin{array}{c}\mathrm{L} \\
\mathrm{n}=12\end{array}$ & $59^{*} \pm 7.07$ & $1727.5^{*} \pm 478.4$ & $26^{*} \pm 8.48$ & $966.48^{*} \pm 377.2$ & $39 * \pm 21.21$ & $16^{*} \pm 2.83$ & $20 \pm 12.1$ & $10 \pm 1.84$ \\
\hline $\begin{array}{c}\text { MA } \\
n=28\end{array}$ & $54.3^{*} \pm 9.62$ & $1589.2^{*} \pm 327.6$ & $30^{*} \pm 8.09$ & $1058.2^{*} \pm 3.46$ & $29.7^{*} \pm 4.85$ & $15.8^{*} \pm 6.12$ & $24.6 \pm 5.46$ & $14.8 \pm 2.16$ \\
\hline $\begin{array}{c}\text { A } \\
\mathbf{n}=\mathbf{3 6}\end{array}$ & $59.6^{*} \pm 12.36$ & $1693.7 * \pm 586.21$ & $29.1^{*} \pm 6.43$ & $1074.1^{*} \pm 302.1$ & $33^{*} \pm 15.52$ & $16.4^{*} \pm 9.62$ & $26.60 \pm 1.2$ & $12.7 \pm 3.42$ \\
\hline $\begin{array}{c}G \\
n=18\end{array}$ & $56.7^{*} \pm 15.14$ & $1662.25^{*} \pm 447.4$ & $29.3^{*} \pm 15.53$ & $1024.2^{*} \pm 296.4$ & $25^{*} \pm 3$ & $14^{*} \pm 4.58$ & $31.70 \pm 9.36$ & $15.3 \pm 6.28$ \\
\hline
\end{tabular}

$\overline{\mathbf{x}} \pm \mathrm{s}$ : média aritmética \pm desvio standar; TBC: L: leve; MA: moderadamente avanzada; A: avanzada; G: grave; RET: rosetas E totales; REA: rosetas $\mathrm{E}$ activas; RETR: rosetas $\mathrm{E}$ teofilina resistentes; $\mathrm{RETS}$ : rosetas $\mathrm{E}^{\circ}$ teofllina sensibles; ${ }^{*} \mathrm{RET}\left(4^{\circ} \mathrm{C}\right)$, REA $\left(37^{\circ} \mathrm{C}\right)$, RETR $\left(4^{\circ} \mathrm{C}\right.$ y $\left.37^{\circ} \mathrm{C}\right)$ significativamente superiores a los correspondientes valores de los pacientes pre-tratamiento (ver Tabla I). $\mathrm{p}<0.01$.

TABLA 3

Relación linfocitos $T$ colaboradores/ $T$ supresores (RETR RETS) de pacientes pre-tratados, tratados y testigos sanos.

\begin{tabular}{llll}
\hline & & \multicolumn{2}{c}{ RETR/RETS } \\
& Condición & $4^{\circ} \mathrm{C}(\overline{\mathrm{x}} \pm \mathrm{s})$ & $37^{\circ} \mathrm{C}(\overline{\mathrm{X}} \pm \mathrm{s})$ \\
\hline L & Pré-tratados & $0.91 \pm 0.25^{*}$ & $0.93 \pm 0.12^{*}$ \\
& Tratados & $1.96 \pm 0.33$ & $1.6 \pm 0.16$ \\
\hline MA & Pré-tratados & $0.65 \pm 0.19^{*}$ & $0.73 \pm 0.11^{*}$ \\
& Tratados & $1.21 \pm 0.30$ & $1.11 \pm 0.12$ \\
\hline & Pré-tratados & $0.86 \pm 0.13^{*}$ & $0.69 \pm 0.09^{*}$ \\
& Tratados & $1.24 \pm 0.12$ & $1.29 \pm 0.10$ \\
\hline G & Pré-tratados & $0.54 \pm 0.3^{*}$ & $0.71 \pm 0.14^{*}$ \\
& Tratados & $0.79 \pm 0.20$ & $0.91 \pm 0.11$ \\
\hline TS & & $1.15 \pm 0.43$ & $1.17 \pm 0.19$ \\
\hline
\end{tabular}

RETR: rosetas E teofilina resistentes; RETS: rosetas E teofilina sensibles; TBC: L: leve; MA: moderadamente avanzada; $A$ : avanzada; $\mathrm{G}$ : grave; $\mathrm{TS}$ : testigos sanos; $\overline{\mathrm{x}} \pm \mathrm{s}$ : média aritmética \pm desvio standar; * Diferencias significativas entre pacientes pre-tratados y tratados $(p<0.01)$ y pre-tratados y TS (p $<0.01$ ).

Los pacientes con baciloscopía (BAAR) altamente positiva, y algunos de ellos anérgicos $O$ con baja respuesta frente a la i.d.r de Mantoux 2 U.T., evidenciaron una marcada deficiencia de células inmunocompetentes. Se demostró asociación entre estas tres variables $(p<0.01)$ Tabla 4.

\section{TABLA 4}

Asociación entre baciloscopia (BAAR), intradermo-reacción de Mantoux (PPD 2 UT) y nivel de celulas inmunocompetentes (NCI) en pacientes tuberculosos virgenes de tratamiento en sus diferentes manifestaciones clinicas.

\begin{tabular}{cccccc}
\hline \multirow{2}{*}{ BAAR } & PPD 2 UT & \multicolumn{5}{c}{ NCI } & No \\
\cline { 3 - 6 } & & $\mathrm{N}$ & $\mathrm{MD}$ & $\mathrm{D}$ & \\
\hline \multirow{2}{*}{1} & $(-)$ & $16.66 \%$ & $44.44 \%$ & $38.88 \%$ & 18 \\
& $(+)$ & $50 \%$ & - & $50 \%$ & 12 \\
\hline 2 & $(-)$ & $30 \%$ & $10 \%$ & $60 \%$ & 16 \\
& $(+)$ & - & - & - & - \\
\hline 3 & $(-)$ & - & $33.33 \%$ & $66.66 \%$ & 16 \\
& $(+)$ & - & $50 \%$ & $50 \%$ & 12 \\
\hline 4 & $(-)$ & $25 \%$ & $25 \%$ & $50 \%$ & 8 \\
& $(+)$ & - & - & - & - \\
\hline
\end{tabular}

BAAR: $1:+, 2:++, 3:+++, 4:++++;$ PPD 2 UT: $(-): 0$ $10 \mathrm{~mm}^{2},(+): 10 \mathrm{~mm}^{2} ; \mathrm{n}:$ número de pacientes; NCI: Porcentaje de individuos que presentó valores normales (N), Medianamen. te Disminuídos (MD) o Disminuídos (D) con respecto a los de los Testigos Sanos.

La evaluación de los niveles de proteínas séricas y los valores de cada una de ellas, en especial el de albúmina, se utilizó para determinar el estado nutricional de los pacientes. Se comprobó que el porcentaje de individuos con un déficit nutricional era superior en los enfermos que en los TS, aunque tal deficiencia en la nutrición no está asociada a la manifestación clínica de la Enfermedad (Tabla 5). 
DLUGOVITZKY, D.; HUBER, K.; WELKER, G. \& MOLTENI, O. - Evaluación de las subpoblaciones de linfocitos $\mathrm{T}$ en pacientes tuberculosos empleando la modulación con teofilina. Rev. Inst. Med. trop. S. Paulo, 33(2): 105-113, 1991.

TABLA 5

Estado nutricional en diferentes manifestaciones de TBC y testigos sanos (TS).

\begin{tabular}{lccccc}
\hline TBC & $\mathrm{L}$ & $\mathrm{MA}$ & $\mathrm{A}$ & $\mathrm{G}$ & $\mathrm{TS}$ \\
$\mathrm{n}=12$ & $\mathrm{n}=28$ & $\mathrm{n}=22$ & $\mathrm{n}=8$ & $\mathrm{n}=15$ \\
\hline Proteínas totales & $\begin{array}{c}5.45 \\
\pm\end{array}$ & $\begin{array}{c}4.9 \\
\pm\end{array}$ & $\begin{array}{c}4.92 \\
\pm\end{array}$ & $\begin{array}{c}5.1 \\
\pm\end{array}$ & $\begin{array}{c}7.59 \\
\pm\end{array}$ \\
$\overline{\mathrm{x}} \pm \mathrm{s}$ & 0.5 & 0.41 & 0.37 & 0.5 & 0.31 \\
\hline Albúmina & 2.72 & 2.46 & 2.52 & 2.4 & 3.7 \\
$\overline{\mathrm{x}} \pm \mathrm{s}$ & \pm & \pm & \pm & \pm \\
& 0.57 & 0.56 & 0.48 & 0.63 & 0.28 \\
\hline
\end{tabular}

TBC: L: leve; MA: moderadamente avanzada; $A$ : avanzada; $\mathbf{G}$ : grave; $\bar{x} \pm \mathrm{s}$ : media aritmética \pm desvio estandar

\section{DISCUSIÓN}

Los datos reportados en este trabajo se suman a los de otros autores que demuestran el amplio espectro de fenómenos supresivos $\mathrm{y}$ fallas en los mecanismos inmunológicos que se ponen en evidencia en los individuos tuberculosos, aunque no siempre es posible relacionarlas a las diversas formas clínicas de la enfermedad.

Nuestros resultados demuestran que los tuberculosos en todas las manifestaciones clínicas cursan con una disminución en el tráfico de células comprometidas en la respuesta inmune celular, que en esta experiencia se pone de manifiesto a través de un descenso en los niveles de linfocitos $\mathrm{T}$ totales, activos y colaboradores.

No se proporcionan en la bibliografia los valores de linfocitos $\mathrm{T}$ totales y activos ni los de la relación $\mathrm{T}$ "helper"/supresores en las diferentes manifestaciones de tuberculosis. Los valores que se informan en este trabajo son el promedio de tales datos, determinados experimentalmente en la población de pacientes estudiada.

La frequencia de pruebas tuberculino-negativas se asoció a la infección masiva o la supresión de la inmunidad que se manifestaría como una disminución de linfocitos $\mathrm{T}$ colaboradores o a un incremento de los linfocitos $\mathrm{T}$ supresores.

Diversas experiencias evidenciaron que la energia frente al test de tuberculina o PPD, que es específica a la respuesta frente a los productos de Mycobacterias en algunos pacientes, puede estar asociada con niveles elevados de anticuerpos anti-Mycobacteria ${ }^{10}$.

Durante el tratamiento del paciente inicial mente anérgico los fenómenos de hipersensibilidad retardada (DTH) se desarrollan y en consecuencia declinan los niveles de anticuerpos.

A través de experimentos in vitro se han demostrado constituyentes mycobacterianos que son potencialmente inmunosupresivos y la existencia de circuitos de células supresoras operantes en enfermos tuberculosos. Es posible dicotomizar los antígenos mycobacterianos en aque llos que activan los mecanismos protectores y los que inducen células supresoras ${ }^{10}$.

Otros experimentos in vitro permitieron evi denciar los mecanismos que resaltan la depresión de las respuestas a tuberculina y $\mathrm{PPD}^{10}$. En pacientes con tuberculosis recientemente diagnosticada, los test tuberculino-negativos han sido asociados con blastogenesis deprimida, inducida por PPD en las células mononucleares en sangre periférica ${ }^{17}$.

Se ha demostrado que las respuestas blastogénicas inducidas por tuberculina están correlacionadas con la producción de interleukina 2 (IL-2). En estos estudios la depleción de células adherentes incrementó la producción de IL-2 ${ }^{17}$.

Por otra parte, en enfermos tuberculosos la depleción de células no adherentes resultó en una producción disminuída de IL-2 ante la estimulación mediada por $\mathrm{PPD}^{17}$.

La protección contra la tuberculosis y la patogénesis de la misma depende en gran medida de células $T$ específicas que mediarían la activación de los macrófagos y a estas poblaciones celulares se las considera las principales responsables de la respuesta del huésped en esta enfermedad.

La restricción del crecimiento celular de ba cilo depende de la interacción entre las diferentes poblaciones celulares de linfocitos y los macrófagos intervinientes ${ }^{10}{ }^{13}, 29$.

ROOK y colab. sugirieron, a través de sus estudios de nódulos linfáticos en tuberculosos 
DLUGOVITZKY, D.; HUBER, K.; WELKER, G. \& MOLTENI, O. - Evaluación de las subpoblaciones de linfocitos T en pacientes tuberculosos empleando la modulación con teofilina. Rev. Inst. Med. trop. S. Paulo, 33(2): 105-113, 1991.

y sujetos normales, la existencia de un atrapamiento de los linfocitos antígeno-reactivos en pacientes con enfermedad severa, especialmente en aquellos con respuesta tuberculínica baja o negativa ${ }^{25}$. Esto explicaría la deficiencia de los linfocitos $\mathrm{T}$ en sangre periférica de los pacientes tuberculosos.

Diversos autores se hallan abocados a lograr nuevos conocimientos de los mecanismos celulares y moleculares operantes en la resistencia del huésped al bacilo tuberculoso $0^{9}$ 14, 10, 20, 23 ya que aún es necesario profundizar lo investigado sobre dichos aspectos.

Mediante estudios in vitro, utilizando anticuerpos monoclonales se ha provocado una depleción selectiva de CD4 y CD8 determinando a la vez las actividades de estas líneas celulares ${ }^{16}$. Se evidenció que en la tuberculosis experimental se generan ambas subpoblaciones CD4 y CD8 y desempeñan un papel protector tanto en los fenómenos que intervienen las células colaboradoras como las citotóxicas.

Estas células expresan su actividad a través de diversos mecanismos contra la patogénesis de la enfermedad ${ }^{16}$.

Se han evaluado sustancias biológicamente activas elaboradas en dicha respuesta celular. Así por ej. se han estudiado las linfoquinas producidas predominantemente en la tuberculosis, que incluyen factores activadores de linfocitos y macrófagos, especialmente interferón $\boldsymbol{\gamma}^{25}$.

En la lesión tuberculosa las linfoquinas producidas por las células $T$ locales causan acumulación y activación de los macrófagos y de linfocitos adicionales que a veces determinan la desaparición de la lesión. Tambien el factor de necrosis tumoral $\boldsymbol{\alpha}$ y el $\boldsymbol{\beta}$ contribuyen al daño tisular hallado en la enfermedad ${ }^{25}$.

La circunstancia de que los pacientes con evolución clínica favorable que evidenciaron capacidad para responder a la quimioterapia, presentando niveles de células inmunocompetentes y en particular células colaboradoras notablemente superiores, permite atribuir al sistema inmune un rol preponderante en la modulación de los fenómenos protectores que se desencadenan ante una infección por bacilo de Koch.
Los resultados de los controles de seguimiento de los enfermos en tratamiento con evolución favorable demostraron una elevación en la relación Rosetas $\mathbf{E}$ Teofilina Resistentes/Rosetas $\mathbf{E}$ Teofilina Sensibles. Se deduce que la cuantificación seriada de células inmunocompetentes permite evaluar la capacidad de protección inmune que se genera en los individuos infectados. Teniendo en cuenta que la técnica empleada no es de excesiva complejidad y brinda datos valiosos para interpretar diversos fenomenos, sería de utilidad como método accesible en el estudio inmunológico y evolutivo de la enfermedad.

En nuestro experimento se verificó también que un porcentaje considerable de los individuos enfermos evidenciaron un déficit nutricional que se puso de manifiesto a través de parámetros tales como proteinemia total y albuminemia, inferiores a los valores obtenidos en los sujetos sanos.

La mal nutrición puede haber sido el factor subyacente de las deficiencias inmunológicas detectadas en algunos pacientes.

Los datos obtenidos permiten concluir que en la tuberculosis pulmonar en sus diferentes formas clínicas (L, MA, A y G) existe una disminución en la cantidad relativa y absoluta de linfocitos $T$, responsables de la respuesta inmune celular. Los valores de Rosetas $\mathrm{E}$ Totales, teofilina sensibles y resistentes (a $4^{\circ} \mathrm{C}$ y $37^{\circ} \mathrm{C}$ ) no difirieron significativamente entre estos grupos de enfermos aunque se evidenció asociación entre la manifestación clínica de la enfermedad y la proporción de linfocitos $\mathrm{T}$ colaboradores. Además los pacientes que presentaron niveles más altos en los controles seriados efectuados después de iniciada la quimioterapia, evolucionaron favorablemente y respondieron al tratamiento.

Esto confirma también que están involucradas ambas poblaciones linfocitarias, tanto colaboradoras como supresoras. La protección que ejercerían las mismas contra la patogénesis de la enfermedad depende de la relación o balance entre estos dos tipos de células $T$.

Se demostró asociación entre la baciloscopía y la respuesta a la i.d.r. de Mantoux y el número de células inmunocompetentes. 
DLUGOVITZKY, D.; HUBER, K.; WELKER, G. \& MOLTENI, O. - Evaluación de las subpoblaciones de linfocitos T en pacientes tuberculosos empleando la modulación con teofilina. Rev. Inst. Med, trop. S. Paulo, 33(2): 105-113, 1991.

También se ha constatado en un elevado número de pacientes un marcado déficit nutricional asociado con la predisposición a contraer la enfermedad.

\section{SUMMARY}

T lymphocyte subsets evaluation in patients with pulmonary tuberculosis using theophylline modulation.

$T$ cells and $T$ cells subsets in peripheral blood of patients with different forms of pulmonary tuberculosis were evaluated to explain some aspects of the immunocompromised state of these subjects.

Diagnosis was made by baciloscopy (BAAR), chest roentgenography i.d.r Mantoux, and other clinical analysis.

Spontaneous E-Rosette test (RE) was used to quantify Total (RET $4^{\circ} \mathrm{C}$ ) and Active T cells (REA $37^{\circ} \mathrm{C}$ ) and the same test after incubation with Theophilline (The) for helper cells (The-resistant cells: RETR) and suppressor cells (Thesensitive cells: RETS). Patients were followed for at least 4 months after therapy.

The data demonstrate a significant decrease of relative and absolute numbers of Total T-cells and a diminished $\mathrm{T}$ helper/T suppressor subset ratio (RETR/RETS) which dropped to less than 1 in untreated patients.

Treated patients with a favourable evolution showed a restoration of Total active and helper T cells. RETR/RETS ratio was also significantly increased.

In patients with highly positive BAAR, low on negative i.d.r Mantoux, a decreased level of immunocompetent cells was observed. The 3 aspects were associated.

Nutritional condition of the patients wal also associated with the predisposition to acquire this disease.

\section{REFERENCIAS BIBLIOGRÁFICAS}

1. ALLEN, E. M.; STERNICK, J.L.; SCHRIER, D. J. \& MOORE, V. L. - BCG induced chronic pulmonary inflamation and splenomegaly in mice: suppression of PHA induced proliferation delayed hypersensitivity to sheep erytrocytes and chronic pulmonar inflammation by soluble factors from adherent spleen cells. Cell. Immunol., 58: 61-71, 1981 .

2. BOOM, W. H.; HUSSON, R. N.; YOUNG, R. A.; DAVID, J. R. \& PIESSENS, W. F. - In vivo and in vitro characterization of murine $\mathrm{T}$ cell clones reactive to Mycobacterium tuberculosis. Infect. Immun., 83: 7013-7117, 1987.

3. COLLINS, F. M. \& CAMPBeLL, S. G. - Immunity to intracellular bacteria. Vet. Immunol. Immunopath., 3: $5-66,1982$

4. COOMBS, R. R. A.; GURNER, B. W.; WILSON, A. B.; HOLM, G. \& LUSCHGRIN, B. - Rosette information bet ween human lymphocytes and sheep red celis not involving immunoglobulin receptors. Int. Arch. Allergy, 39: 658-665, 1970

5. DANIEL, T.M. - The immunology of tuberculosis. Clin Chest Med., 1: 189-201, 1980

6. DANIEL, T. M.; OXTOBY, M. J.; PINTO, R. \& MORENO E. - The immune spectrum in patients with pulmonary tuberculosis. Amer. Rev. resp. Dis., 123: 556-559, 1981.

7. DANNEMBERG, A. M. - Pathogenesis of tuberculosis: native and acquired resistence in animals and humans microbiology. Washington, American Society for Microbiology, 1984. p. 344-354

8. DANNEMBERG, JR, A. M. - Pathogenesis of pulmonary tuberculosis. In: FISHMAN, A. P., ed. Pulmonary diseases and disorders. 2 nd. ed. New York, Mac Graw-Hill, 1988. p. $1821-1842$.

9. DE LIBERO, G.; FLESCH, I. \& KAUFMANN, S. H. E - Mycobacteria reactive Lyt $2{ }^{+}$T-cell lines. Europ. J. Immunol., 18: 59-66, 1987.

10. ELLNER, J. J. \& WALLIS, R. S. - Immunologic aspects of mycobacterial infections. Rev. infect. Dis., 11 (Suppl. 2): S455-S459, 1989

11. ESTRADA PARRA, S. - La respuesta inmunológica en la tuberculosis. Salud publ. Méx., 25: 403-409, 1983.

12. FUJIWARA, H.; OKUDA, T.; FUKUKAWA, T. \& TSUYOGUCH, I. - In vitro tuberculin reactivity of lymphocyte from patients with tuberculosis pleuresy. Infect. Immun. 35: 402-409, 1982

13. HAHN, H. \& KAUFMANN, S. H. E. - The role of cell-me diated immunity in bacterial infections. Rev. infect. Dis., 3: $1221-1250,1981$

14. KAUfmanN, S. H. E. \& FLESCH, I. - Function and antigen recognition pattern of $L_{3} T, T$-cells clones from Mycobacterium tuberculosis immune mice. Infect. Im mun., 54: 291-296, 1986

15. KAUFMANN, S. H. E.; VATH, V.; THOLE, J. E. R.; VAN EMBDEN, J. D. A. \& EMMRICH, F. - Enumeration of $T$ celis reactive with Mycobacterium tuberculosis organisms and specific for the recombinant mycobacterial 64-KDa protein. Europ. J. Immunol., 17: 351-357, 1987. 
DLUGOVITZKY, D.; HUBER, K.; WELKER, G. \& MOLTENI, O. - Evaluación de las subpoblaciones de linfocitos T en pacientes tuberculosos empleando la modulación con teofilina. Rev. Inst. Med. trop. S. Paulo, 33(2): 105-113, 1991.

16. KAUFMANN, S. H. E. - In vitro analysis of the cellular mechanisms involved in immunity to tuberculosis. Rev. infect. Dis., 11 (Suppl. 2): S448-\$459, 1989.

17. KLEINHENZ, M. E. \& ELLNER, J. J. - Antigen responsiveness during tuberculosis: regulatory interactions of $T$ cell subpopulations and adherent cells. J. Lab. clin. Med., 110: 31-40, 1987.

18. LAMB, J. R.; IVANYI, J.; REES, A.; YOUNG, R. A. \& YOUNG, A. B. - The identification of $T$ cells, epitopes in Mycobacterium tuberculosis using human $T$ lymphocyte clones. Leprosy Rev., 57: 131-137, 1986.

19. LIMAMBUL, S.; SHORE, A.; DOSCH, N.M. \& GELFAND W. - Theophylline modulation of $E$ rosette formations: an indicator of $\mathrm{T}$-cell maturation. Clin. exp. Immunol. 33: $503-508,1978$

20. MÜLLER, I.; COBBOLD, S. P.; WALDMAN, H. \& KAUF MANN, S. H. E. - Impaired resistance to Mycobacterium tuberculosis infection after selective in-vivo depletion of $L_{3} T_{4}$ and Lyt $2^{+} T$ cells. Infect. Immun., 55: 2037-2041, 1987 .

21. OfTUNG, F.; MUSTAFA, A. S.; HUSSON, R. A. \& GODAL, T. - Human $T$ cell clones recognize two abundant Mycobacterium tuberculosis protein antigens expressed in Escherichia coli. J. Immunol., 138: 927-931, 1987.

22. ORME, I. A. \& COLLINS, F. M. - Adoptive protection of Mycobacterium tuberculosis infected lung: dissociation between cells that passively transfer protective immunity and those that transfer delayed-type hipersensitivity to tuberculin. Cell. Immunol., 84: 113-120, 1984

23. ORME, I. M. - The kinetics of emergence and loss of mediator $T$ lymphocytes acquired in response to infection with Mycobacterium tuberculosis. J. Immunol., 138: 293-298, 1987.

24. PIESSENS, W. F. - Introduction to the immunology of tuberculosis. Rev. infect. Dis., 11 (Suppl. 2): S436-\$441, 1989 .
25. ROOK, G. A. W. - Role of activated macrophages in the immunology of tuberculosis. Brit. med. Bull., 44: $611-623,1988$.

26. ROOK, G. A. W.; CARSWELL, J. W. \& STANFORD, J. J. - Preliminary evidence for trapping of antigen specific lymphocytes in the lymphoid tissue of anergic tuberculosis patients. Clin. exp. Immunol., 26: 129-132, 1976.

27. SHOAT, B. \& JOSHNA, H. - Supressor helper and immunoregulatory $\mathrm{T}$ cells in normal human biood as defined by theophylline sensitivity. Thymus, 4: 323, 1982

28. SMITH, D. W. \& WEIEGESHAUS, E. H. - What animal models can teach us about the pathogenesis of tuberculosis in man. Rev. infect. Dis., 11 (Suppl. 2): \$385-\$393, 1989 .

29. STEEL, J.; FLINT, K. C.; POZNICK, A. L.; HUDSPITH, B.; JOHNSON, M. M. \& ROOK, G. A. W. - Inhibition of virulent Mycobacteria tuberculosis by murine peritoneal macrophages and human alveolar lavage cells. The effect of lymphokines and recombinant gamma interferon. Tubercle, 67: 289-294, 1986.

30. VRIES, R. R. P. - Regulation of T cell responsiveness against mycobacterial antigens by HLA class. 2 immune response genes. Rev. infect. Dis., 11 (Suppl. 2): $\$ 400$ S403, 1989.

31. WYBRAM, J. - The human rosette forming cells as a marker of a population of thymus derived cells. J. clin. Invest., 51: 2537-2545, 1972.

32. WYBRAM, J. - Rosette forming cells in immunologic def ficience diseases and transferfactor. New Engl. J. Med. 281: 710-716, 1973.

Recebido para publicacão em 21/6/1990 Aceito para publicação em 1/3/1991. 